\title{
The Association Between Residential Proximity to A Major Roadway and the Prevalence of Environmentally Influenced Conditions
}

\author{
Daniel C. Indorato* \\ College of Medicine, American University of Antigua, Coolidge, Antigua, USA
}

*Corresponding Author: Daniel C. Indorato, College of Medicine, American University of Antigua, Coolidge, Antigua, USA, Tel: (978)-979-3396; E-mail: DanielIn@auamed.net

Received: 01 February 2018; Accepted: 13 February 2018; Published: 15 February 2018

\begin{abstract}
Background: Motor vehicles are the prime culprit of air-pollution, and could be possibly linked to the development of widely evident health hazards.
\end{abstract}

Objective: The purpose of the current study was to examine the association between residential proximity to a major roadway and the prevalence of metabolic disorders, respiratory diseases, and stress levels.

Materials and Methods: A cross-sectional survey was administered to 99 randomly selected subjects (Males: 33, Females 66) aged between 18 and 74 years, who lived at a residence for a minimum one to five years.

Results: The findings in the current study reveal an increase in the prevalence of anxiety, depression, asthma, allergies, attention deficit disorder, type 2 diabetes, hypertension, and self-reported stress seen in individuals who live within five miles of a major highway. A negative correlation was observed between residential distance from a major highway and the prevalence of asthma/allergies and type 2 diabetes.

Keywords: Air pollution; Highways; Disease; Depression; Anxiety; Stress

\section{Introduction}

The earth's climate has been rapidly changing, mainly due to the large amounts of greenhouse gas emissions as a result of human activity. These gas emissions are derived from transportation systems, infrastructure, and building 
construction [1]. Urban air pollution is a major pollution concern and environmental risk that place a heavy burden on the world's population [16]. Previous research suggests that physical and mental health problems are correlated with the surrounding environment such as homes, schools, workplaces, parks, industrial areas, farms, roads and highways [18].

Motor vehicles are a leading source of air pollution [2]. Motor vehicles are also a major source of other greenhouse gases that include methane, nitrogen oxides, and volatile organic compounds [2,4]. The health hazards of air pollution are widely evident, especially due to the irritant effects of ozone [3]. Higher ozone levels are associated with higher incidence and severity of respiratory symptoms, worse lung function, increased emergency room visits and hospitalizations, more medication use, and more absenteeism from school and work [3]. Ozone and other harmful compounds present in the air due to motor vehicles contribute to human health problems such as lung disease [5]. The people most affected by air pollution include older adults with preexisting respiratory disease, children, especially those with asthma, individuals with inadequate health care, and even healthy individuals who work or exercise outdoors [6,7].

A study conducted in the city of Sao Jose dos Campos, Paraiba Valley demonstrated a significant relationship between air pollutants and an increased number of asthma related hospitalizations among children up to 10 years of age [8]. Toledo et al. implemented a cross-sectional study of adolescents in order to evaluate the temporal trends in the prevalence of asthma, rhinitis, and eczema in adolescents who live in Taubate, Brazil. The researchers demonstrated an increase in the prevalence of these diseases as the residential proximity to a heavily travelled highway decreased. The results of this study indicated that a higher frequency of asthma and rhinitis is seen among adolescents living close to the heavily travelled highway.

Depression, which is a common cause of morbidity, is major health problem. Depression is commonly associated with an imbalance of brain chemicals, triggered by stress, and a combination of biological, psychological, and social factors, in addition to air pollution. A time-series study in Edmonton, Canada examined and assessed the associated between emergency department (ED) visits for depression and air pollution. In this analysis, the researchers found a positive correlation between ED visits for depression and exposure air pollution [19].

Air pollution is associated with an increased risk for cardiovascular events [11]. Studies have revealed convincing evidence that individuals with diabetes are more susceptible to cardiovascular health effects associated with air pollution [12, 13, 23]. Kramer et al. implemented a cohort study based on cross-sectional surveys administered to nondiabetic women who were 54-55 years old at baseline. The indications of this study were the utilization of a large, specific sample size, as well as the long-duration of the study which was assessed over a 16-year span. After the 16-year incidence analysis, researchers found that traffic-related air pollution was associated with incidence of type 2 diabetes among elderly women. The current study can be distinguished from the cohort study proposed by Kramer et al. by the wide age group covered. These criteria make the current cross-sectional analysis more generalizable to the population [24]. 
The current literature has investigated the associations between air pollution and noise exposure with impaired neurocognitive functioning [21]. A review from Tzivian et al. identified articles related to the long-term effects of air pollution and ambient noise in association with cognitive functioning, verbal and non-verbal learning, memory, anxiety, and depression. Both exposures were shown to be associated with elevated anxiety and delayed cognitive functioning. While the evidence from this review suggests that an association exists between anxiety and exposure to pollution, the results are ineffective to generate an overall conclusion about the independent effect of prolonged pollutant exposure. The scare literature with regards to examining the association between self-reported stress and geographical data, prompted the researchers interest towards the current investigation [21].

To our knowledge, no one has attempted a cross-sectional study to investigate a possible link between self-reported stress, anxiety, depression with exposure to air pollution, based on their geographical location. The lack of studies investigating the effects of residentially proximity to a heavily travelled roadway with the prevalence of multiple organ system diseases (type 2 diabetes, hypertension, depression, anxiety etc.) other than respiratory conditions (asthma, chronic obstructive pulmonary disease, etc.), helps separate the current research from previous literature. Therefore, the researcher believes that the findings in the current cross-sectional analysis will aid in the development of further investigations that are directed towards the prevention and maintenance of these conditions.

\section{Materials and Methods}

\subsection{Study Subjects}

This epidemiological cross-sectional study was conducted at American University of Antigua College of Medicine. The study included a total of 99 randomly selected subjects (Males: 33, Females 66) aged between 18 and 74 years, who lived at a residence for a minimum one to five years. The subjects were asked to answer a Standardized Disease Prevalence Questionnaire (SDPQ) which was designed to assess the distance that each subject lived from a major highway in relation to the prevalence of certain diseases/conditions within each household. The participants signed informed consent forms before their inclusion in the study. The sample size was calculated based on $80 \%$ power, $5 \%$ alpha error, and a $4 \%$ confidence interval, which resulted in a calculated sample size of 250 subjects. However, after the exclusion criteria was applied to each participant, the researcher was able to collect a sample size of 99 subjects. As a result, the final power of the study was calculated to be $47.5 \%$. The present study included a broad age range, demographic population, and was not limited to a specific gender. The great degree of variation in the current sample was designed to enhance the external validity of the study, in comparison to previous studies that have focused on assessing the effects of highways in specific populations from poorly developed countries [6, 10, 14, 20].

\subsection{Data Collection}

The SDPQ was a ten-item questionnaire, in which the first three questions of the were standardized demographic questions that included Gender, Age, and Ethnicity respectively. According to the answers to the SDPQ, subjects were classified into three groups: living within $0.1-1$ mile of a major highway, $1.1-4.9$ miles, or 5.0+ miles. The criteria the researchers used to determine a "major highway" was the nearest roadway named as either an expressway, highway, turnpike, interstate, freeway. In order to assess each distance, Question four asked the subjects 
to provide their State, City, and Street name. Geographic information software (ArcGIS, Version 10.2) was used to compute the distance from the residential address of each participant to the nearest US census class code A1 roadway. Class code A1 road segment was defined as a roadway having limited access to primary roads with defined exits. Question five asked participants; "How long have you resided at the address listed above?” Participants were classified into four categories: Less than 1 year, 1-5 years, 5-10 years, 10+ years. Subjects who resided at their listed address for less than one year were not included in this study. Question six asked; "Do you suffer from any of the following ailments? (Select all that apply)” Answers included Allergies, Anemia, Anxiety disorders, Asthma, Attention deficit disorder, Bipolar disorder, Chronic pain, Chronic Obstructive Pulmonary Disease, Crohn's Disease, Deep-vein Thrombosis, Depression, Gastrointestinal Disorders, High cholesterol, Hyperthyroidism, Hypertension, Rheumatoid arthritis, Type 1 and Type 2 Diabetes. Question seven asked how long subjects have suffered from each condition. Question eight asked if anyone in the same household suffers from any of the same ailments provided in question six, while question nine asked how long the additional member of the household suffered from the selected condition. Question ten of the SDPQ was used to assess the correlation between the prevalence of self-reported stress with residential distances from highways. The question asked; "How bothered do you feel by emotional problems such as feeling anxious, depressed irritable, or sad?”

\subsection{Statistical Analysis}

The data was expressed as percentages Data were collected from August to December 2017. The data were entered in a standard spreadsheet (Excel) and then transferred to SPSS 20.0 software for statistical analysis. The correlation analyses were determined by the Pearson correlation test. Statistical significance was considered to be $P<0.05$.

\section{Results}

The subjects in the current study were predominantly female (66.6\%). Of these individual, most were White/Caucasian (65.6\%) or Hispanic (14.1\%). The majority of the sample was found to be within the age range of 18-24 (40.4\%), while $35.4 \%$ of the subjects were classified within the age range of 25-34.

The most prevalent self-reported conditions were allergies (40.8\%), anxiety disorders (26.5\%), asthma (20.0\%), depression (19.0\%), high cholesterol (31.6\%), and hypertension (22.1\%). Figure 1 shows the distribution of subject's responses that have identified themselves or another individual residing in the same household as having allergies/asthma, depression, anxiety, type 2 diabetes, attention deficit disorder, and hypertension. The prevalence of the remaining conditions that were asked in SDPQ did not demonstrate any significance between each of the three residential distance classifications. $24 \%$ of the sample who lived far from an A1 road segment (5.0+ miles), exhibited a low prevalence of each condition in comparison to subjects living within five miles of a major roadway. The combination of allergies and asthma was the most frequently reported by all subjects, especially for those who lived within one mile of an A1 road segment compared to subjects in the long-distance category (5.0 - 10 miles) (70.0\% vs. 59.0\%). This same pattern was also observed for the prevalence of type 2 diabetes (34.0\% vs 9.1\%), hypertension (27.0\% vs $9.0 \%$ ), depression ( $52.0 \%$ vs. $9.5 \%$ ), and anxiety (48.0\% vs $13.6 \%$ ). 
Figure 2 represents Question ten, showing the distribution of the subject's responses in which they classified themselves as being very bothered, somewhat bothered, or not at all bothered by acute stressors. A pattern was observed in the very bothered category, which demonstrated a significant difference in the self-reported stress levels to an acute stressor ( $55.0 \%$ vs $0.0 \%$ ). No subjects in the long-distance category ( 5.0 miles -10 miles) reported that they were very bothered by emotional problems, and did not report feeling as anxious, depressed irritable, or sad.

In Figure 3, Pearson correlation analysis showed a significant negative correlation between allergies/asthma and residential distance from a major highway (Pearson $r^{2}=0.99725 ; P=<0.001, \mathrm{n}=91$ ). The line represents the linear regression of data $(y=-5.5 x+75.333)$. In Figure 4 , Pearson correlation analysis showed a significant negative correlation between type 2 diabetes and residential distance from a major highway (Pearson $r^{2}=0.83603 ; P=$ $<0.001, \mathrm{n}=91)$. The line represents the linear regression of data $(\mathrm{y}=-0.1245 \mathrm{x}+0.4327)$.

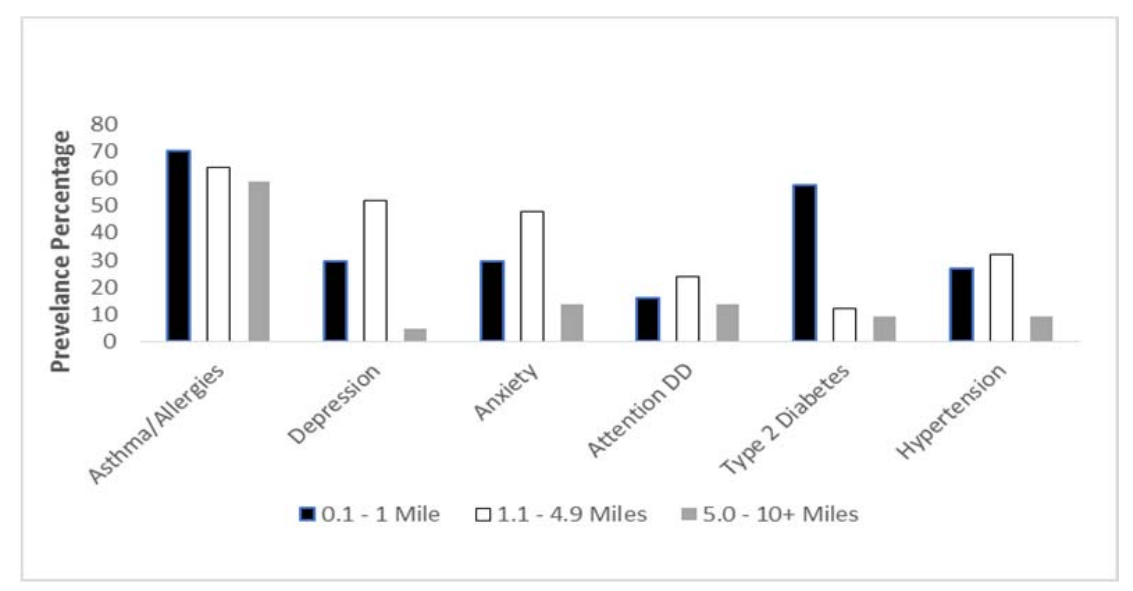

Figure 1: Prevalence of diseases in relation to residential distance from a major roadway

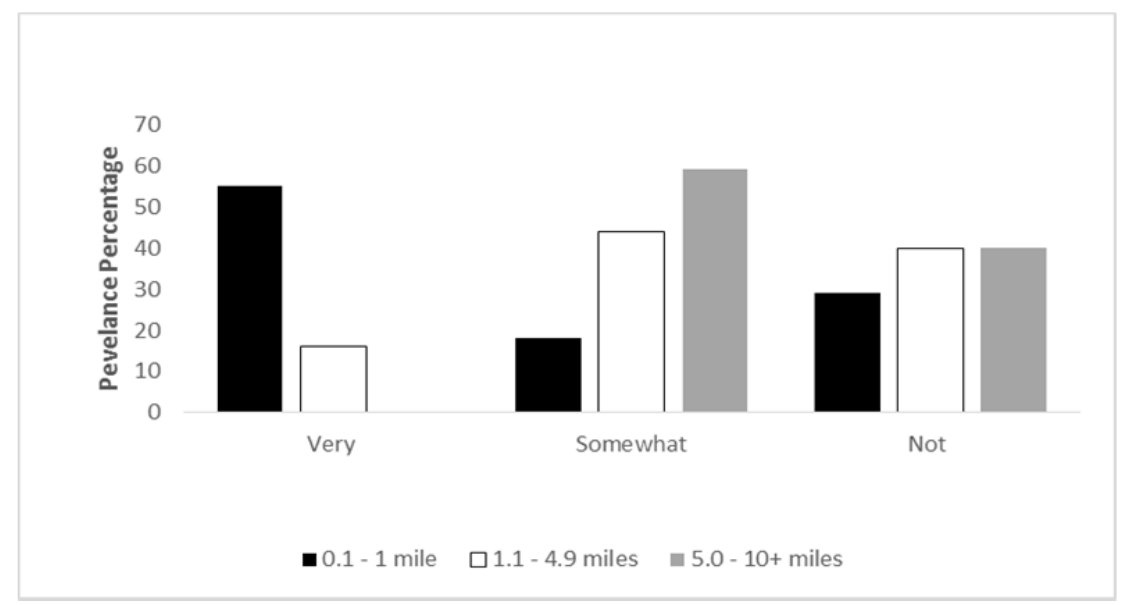

Figure 2: Self-reported stress levels, classified as being very bothered, somewhat bothered, or not at all bothered by emotional problems in relation to residential distance from a major roadway 


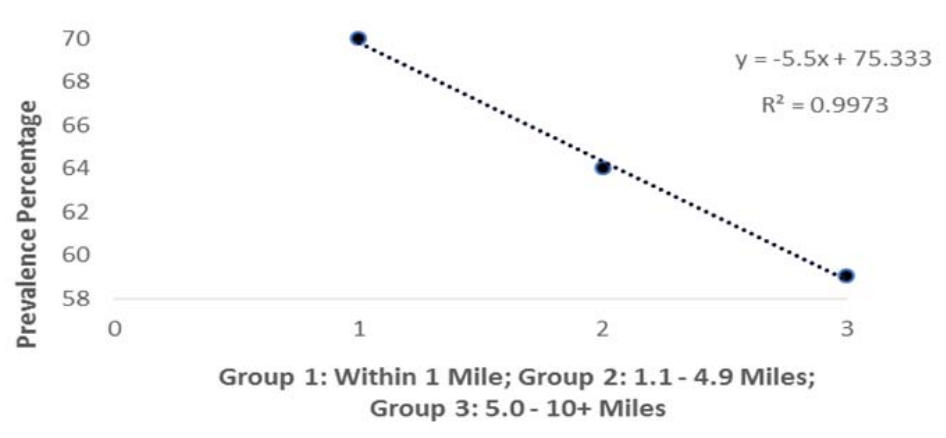

Figure 3: Pearson correlation analysis of asthma/allergies prevalence with residential proximity to a major roadway

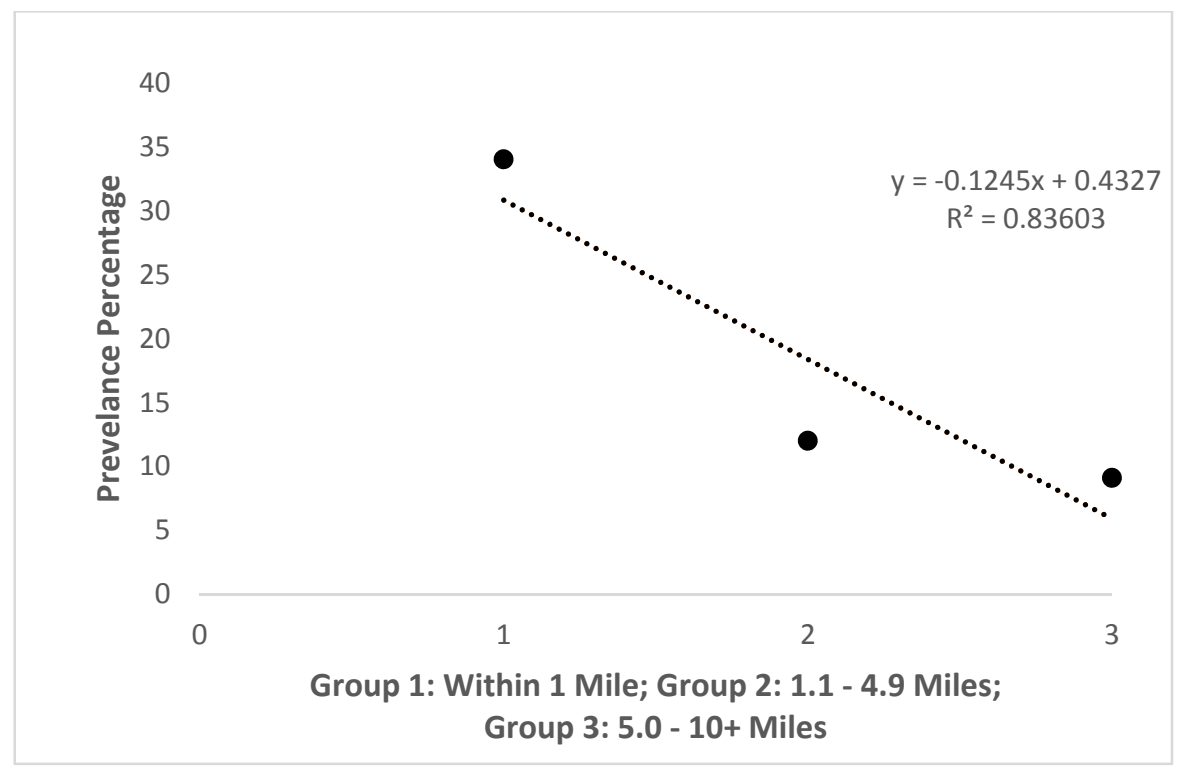

Figure 4: Pearson correlation analysis of type 2 diabetes with residential proximity to a major roadway

\section{Discussion}

To the best of our knowledge, this is the first cross-sectional investigation that shows a significant correlation between residentially proximity to highways and the prevalence of respiratory disease, metabolic disorders, and selfreported stress. This cross-sectional study analyzes the prevalence of various adverse health conditions with respect to each subject's residential proximity to a major roadway in 2017. The present study demonstrates an increase in the prevalence of: asthma, allergies, type 2 diabetes, anxiety, depression, attention deficit disorder, hypertension, and stress levels in subjects who lived within five miles of a major roadway (Figure 1, 2). The prevalence of these conditions was compared to individuals who live a distance that is greater than five miles form a major highway. Participants who lived greater than five miles from a major roadway had a lower prevalence in asthma/allergies, attention deficit disorder, type 2 diabetes, hypertension, anxiety, and depression. There was also a significant difference in the prevalence of self-reported stress in which subjects identified themselves of being "very bothered" 
by emotional problems (Figure 2). Certain combinations of physical and social environmental exposures might produce fluctuations in an individual's stress levels. Exposure to substances form traffic-related air pollution such as nitrogen dioxide, has been linked to the development of chronic stress [17]. These interactions have also showed that higher levels of stress in correlation to increased nitrogen dioxide concentrations, may be associated with chronic inflammation [17]. Self-reported stress levels from the SDPQ in the current study have demonstrated that a significant correlation exists between residentially proximity and traffic-related air pollution. After performing the data analysis, the researcher was unable to detect whether a significant difference exists with respect to the demographical data and the prevalence of diseases in each group. This data was collected in the first three questions of the SDPQ.

The results provide more convincing evidence that exposure to local air pollution from traffic may increase the risk of respiratory disease, stress, and environmentally influenced metabolic disorders (type 2 diabetes, hypertension, etc.). Emerging evidence also suggests that exposure to traffic-related air pollution may be associated with an increased risk of autism during gestation [22]. Due to the findings from this study, it may be reasonable to conclude that pregnant women should take preventative measures which reduce their exposure to traffic-related air pollution. In summary, the current findings provide further indication to emphasize the need for families to become increasingly aware of their surrounding environments, and to adopt the necessary precautionary behaviors which limit exposure to motor vehicle emissions for both themselves and their developing newborn.

While the researchers were not able to obtain data regarding the toxic emissions from the heavy traffic on each roadway, it was hypothesized that the significant differences in prevalence between each classified distance could be attributed to traffic-related air pollution. The prevalence of asthma and allergies has undergone extensive investigation throughout the world. However, there is great uncertainty regarding the reasons for the variability in disease prevalence. Genetic, phenotypic, and environmental factors could potentially have an influence on the variability in these diseases $[9,10]$. Therefore, research which excludes subjects that have a genetic predisposition to asthma, or certain allergies, will give a better estimate of the influence of exposure to toxic emissions from motor vehicles.

The main limitation of this study is the fact that the data obtained was self-report only. This limitation may have led to the misclassification of subjects through the failure to report the presence of a certain condition. However, there is a high reliability associated with these reports when compared to previous studies that has explored the associations between traffic-related air pollution and the current conditions under investigation in the current study. Although the data in this cross-sectional analysis is consistent with the current literature, the researchers acknowledge the limitations of self-report data and believe that future studies which investigate the current research question should implement new test methods that improve both data collection and analysis. Such work should focus on reducing error through appropriate study design and statistical methods [15]. 
Another limitation of the present study is that other environmental factors could have contributed to the significant differences observed in the current study. Due to the limited data that was obtained from the SDPQ, the researcher was unable to obtain a full comprehensive medical history from each subject. Therefore, subjects who may have had a genetic predisposition for a certain disease, without any association with environmental factors, could have altered the prevalence data collected from certain conditions. Further research is still needed to investigate the extent to which air pollution caused by motor vehicle emissions affects the overall health of local citizens within close proximity to traffic-related air pollution. The small sample size of this cross-sectional analysis may have affected the significant differences between each classified group. The majority of the sample resides within one mile of a major roadway. Having a larger sample of individuals living within close proximity to traffic-related air pollution may have increased the prevalence of each self-reported condition. Therefore, additional research which utilizes proper study design methods by having an equal distribution of subjects in each group is required. The small sample was also a limiting factor of this research study. The inclusion criteria for the SDPQ, which required subjects to reside at their current location for at least five years, resulted in a substantial loss of potential subjects. However, our sample size was larger compared to those seen in similar studies [17].

\section{Further research}

In order to promote research regarding the findings in the current study, the authors believe that future studies should focus on: developing efficient protocols for sustainable communities, conducting research on the positive health impacts of sustainable communities, assessing the relationships of environmentally influenced health-related conditions in communities that utilize sources of alternate energy (for transportation, agriculture, etc.). There is also a need for further investigation into air pollution and noise exposure in association with mental health. The current literature supports a positive correlation between environmental factors and mental health, but this relationship is not sufficient enough to formulate an overall cause and effect conclusion between the two variables.

\section{Conclusion}

The findings in the current study reveal an increase in the prevalence of anxiety, depression, asthma, allergies, attention deficit disorder, type 2 diabetes, hypertension, and self-reported stress seen in individuals who live within five miles of a major highway. A negative correlation was observed between residential distance from a major highway and the prevalence of asthma/allergies and type 2 diabetes. The prevalence of Type 2 Diabetes decreased as the residential distance form a major highway increased. A similar correlation was also observed in the subject's responses to their reactions when encountering emotional distress. While the current study is unable to demonstrate a cause and effect relationship, further research is still needed in order to focus on particular environmental irritants that could potentially be associated with the onset and progression of these conditions.

\section{Ethical disclosures}

Confidentiality of data. The authors declare that they have followed the protocols of their work and that all the patients included in the study have received sufficient information and have given their informed consent in writing to participate in the study. 


\section{Right to privacy and informed consent}

The authors must have obtained the informed consent of the patients and/or subjects mentioned in the article. The author for correspondence must be in possession of this document.

\section{Conflict of interest}

The authors have no conflict of interest to declare.

\section{References}

1. Younger M, Morrow-Almeida HR, Vindigni SM., Dannenberg AL. The Built Environment, Climate Change, and Health: Opportunities for Co-Benefits. American Journal of Preventive Medicine 35 (2008): 517-526.

2. National Assessment Synthesis Team. Climate change impacts on the United States: the potential consequences of climate variability and change. New York: Cambridge University Press; 2000

3. Committee of the Environmental and Occupational Health Assembly, American Thoracic Society. Health effects of outdoor air pollution. Am J respire Crit Care Med 153 (1996): 3-50.

4. Patz JA, Engelberg D, Last J. The effects of changing weather on public health. Annu Rev Public Health 21 (2000): 271-307.

5. Frumkin H. Urban sprawl and public health. Public Health Rep 117 (2002): 201-217.

6. Spannhake EW, Reddy SP, Jacoby DB, Yu XY, Saatian B, Tian J. Synergism between rhinovirus infection and oxidant pollutant exposure enhances airway epithelial cell cytokine production. Environ Health Perspect 110 (2002): 665-670.

7. McConnell R, Berhane K, Gilliland F, et al. Asthma in exercising children exposed to ozone: a cohort study. Lancet 359 (2002): 386-391.

8. Amâncio CT, Costa Nascimento LF. Asthma and ambient pollutants: a time series study. Rev Assoc Med Bras 58 (2012): 302-307.

9. Vercelli D. Discovering susceptibility genes for asthma and allergy. Nat Rev Immunol 8 (2008): 169-182.

10. Farrokhi S, Gheybi MK, Movahhed A, Dehdari R, Gooya M, Keshvari S, et al. Prevalence and risk factors of asthma and allergic diseases in primary schoolchildren living in Bushehr, Iran: phase I, III ISAAC protocol. Iran J Allergy Asthma Immunol 13 (2014): 348-355.

11. Miller KA, Siscovick DS, Sheppard L, Shepherd K, Sullivan JH, Anderson GL, et al. Long-term exposure to air pollution and incidence of cardiovascular events in women. N Engl J Med 356 (2007): 447-458.

12. O’Neill MS, Veves A, Zanobetti A, Sarnat JA, Gold DR, Economides PA, et al. Diabetes enhances vulnerability to particulate air pollution-associated impairment in vascular reactivity and endothelial function. Circulation 111 (2005): 2913-2920.

13. Krämer U, Herder C, Sugiri D, et al. Traffic-Related Air Pollution and Incident Type 2 Diabetes: Results from the SALIA Cohort Study. Environmental Health Perspectives 118 (2010): 1273-1279. 
14. Porebski G, Wózniak M, Czarnobilska E. Residential proximity to major roadways is associated with increased prevalence of allergic respiratory symptoms in children. Ann Agric Environ Med 14 (2014): 760766.

15. Subar AF, Freedman LS, Tooze JA, et al. Addressing Current Criticism Regarding the Value of Self-Report Dietary Data. The Journal of Nutrition 145 (2015): 2639-2645.

16. Bickerstaff K, Walker G. Public understandings of air pollution: the 'localisation' of environmental risk. Global Environmental Change 11 (2001): 133-145.

17. Chen E, Schreier HMC, Strunk RC, Brauer M. Chronic Traffic-Related Air Pollution and Stress Interact to Predict Biologic and Clinical Outcomes in Asthma. Environmental Health Perspectives 116 (2008): 970975.

18. Srinivasan S, O'Fallon LR, Dearry A. Creating Healthy Communities, Healthy Homes, Healthy People: Initiating a Research Agenda on the Built Environment and Public Health. American Journal of Public Health 93 (2003): 1446-1450.

19. Szyszkowicz, M. Air Pollution and Emergency Department Visits for Depression in Edmonton, Canada. International Journal of Occupational Medicine and Environmental Health 20 (2007): 241-245.

20. Toledo MF, Saraiva-Romanholo BM, Oliveira RC, Saldiva PHN, Silva LFF, Nascimento LFC, Solé D. Changes over time in the prevalence of asthma, rhinitis and atopic eczema in adolescents from Taubaté, São Paulo, Brazil (2005-2012): Relationship with living near a heavily travelled highway. Allergologia et Immunopathologia 44 (2016): 439-444.

21. Tzivian L, Winkler A, Dlugaj M, Schikowski T, Vossoughi M, Fuks K, Hoffmann B. Effect of long-term outdoor air pollution and noise on cognitive and psychological functions in adults. International Journal of Hygiene and Environmental Health 218 (2015): 1-11.

22. Volk HE, Lurmann F, Penfold B, Hertz-Picciotto I, Mcconnell R. Traffic-Related Air Pollution, Particulate Matter, and Autism. JAMA Psychiatry 70 (2013): 71.

23. Oneill M, Veves A, Zanobetti A, et al. Diabetes Enhances Vulnerability To Particulate Air PollutionAssociated Impairment In Vascular Reactivity And Endothelial Function. Epidemiology 15 (2004): S49.

24. Krämer U, Herder C, Sugiri D, et al. Traffic-Related Air Pollution and Incident Type 2 Diabetes: Results from the SALIA Cohort Study. Environmental Health Perspectives 118 (2010):1273-1279.

Citation: Daniel C. Indorato. The Association Between Residential Proximity to A Major Roadway and the Prevalence of Environmentally Influenced Conditions. Archives of Clinical and Biomedical Research 2 (2018): 021-030.

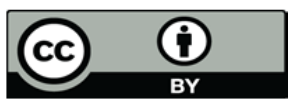

This article is an open access article distributed under the terms and conditions of the Creative Commons Attribution (CC-BY) license 4.0 